\title{
Performance Evaluation of DSDV Routing Protocol with CSMA/CA Strategy in Ad Hoc Network
}

\author{
Samar T. AL-Ani \\ Department of Software Engineering / Baghdad College of Economic Sciences University, Iraq
}

\begin{abstract}
An ad hoc network is a wireless network that does not depend on fixed wired infrastructure. And because of the limited range of packet transmission and reception in a wireless network, the routing protocol is used to expedite communication within the network by finding optimal route between source and destination nodes. In this paper we evaluate Destination-Sequenced Distance-Vector Routing (DSDV) which is a proactive routing protocol where each node maintains routing information for all known destinations, and including CSMA/CA protocol along with RTS/CTS exchanges mechanism to increase the number of successful sending packets and decrease collisions within WLAN, This method achieves strong performance in terms of throughput especially in crowded networks.
\end{abstract}

Keywords: MANET, Ad hoc, DSDV, CSMA/CA, RTS/CTS

\section{Introduction}

A MANET is formed by a group of wireless nodes, each of which performs routing functions and forwards packets for others. No fixed infrastructure (i.e., an access point) is required, and wireless nodes are free to move around. A fixed infrastructure can be expensive, time-consuming, or impractical. Another advantage of MANETs is the expansion of communication distance. In an infrastructure wireless network, nodes are restricted to move within the transmission range of access points. Ad hoc networks relax this restriction by cooperative routing protocols where every node forwards the packet for the rest of the nodes in the network. Potential applications of wireless ad hoc networks include military battle field, emergency rescue, campus networking, etc. [1]

Due to a dynamic nature of ad hoc networks, traditional fixed network routing protocols are not viable. Based on that reason several proposals for routing protocols have been presented. Destination-Sequenced-Distance-Vector (DSDV) is a table-driven routing protocol. Each node's table contains all the network existing destinations, the next hop for every destination, and a metric that indicates the cost of the route. Also, each destination has a sequence number, indicating how old a route is. When a route update with a higher sequence number is received, it replaces the old route. In the case of different routes with the same sequence number, the route with the better metric is used. Updates have to be transmitted periodically or immediately when any significant topology change is detected. [2]

In recent years, the fast increasing demand for high-speed wireless internet access motivated researchers to make efforts for improving the efficiency of decentralized wireless networks[3] [4]. The traditional single band CSMA/CA system has the advantage of requiring neither signaling for bandwidth request nor planned allocation. Request To Send / Clear To Send (RTS/CTS) mechanism should be along with the CSMA/CA channel contention mechanism. The $\mathrm{RTS} / \mathrm{CTS}$ operation is used to solve common wireless problems such as collision. [5]

\section{Literature Survey}

A lot of refinement has been done in MAINT technology along with new proposals for routing protocols. One of the most known routing protocols is (DSDV) which is suited for a multi-hop ad-hoc network is analyzed in [6]. Taking into consideration demands that affect traditional routing protocols (such as loop free routing, completeness, and stability) for delivering data packet during routing in MANET was discussed in [7] [8]. Destination-SequencedDistance-Vector (DSDV) was suggested as a solution for these issues to develop ad hoc routing protocols [9], which is not so much concerned with energy consumption as it is table driven. There has been a lot of research done for the improvement of the proactive as well as reactive protocols [10] [11].

In [12] author proposed a routing tree as an Energy-Efficient Traffic-Aware Detour Tree to solve the problems of link breakage, congestion and energy consumption as the Quality of service routing is challenged in the normal routing strategy. The problems of stale routes and larger size network having a larger number of nodes were overcome in [13] using Eff-DSDV, where author have devised an algorithm indicating that the performance evaluation of the given solution is superior as compared to original DSDV under the performance evaluation parameters. In [14] author has proposed an approach to energy conservation and reducing packet storming within the routing protocol of the ad-hoc network by the use of sending and receiving sleep mode sleep and wake up message.

CSMA/CA was chosen as the core media access mechanism and proposed in [15] to schedules a node to transmit at a specific time slot according to the signal-to-noise-ratio (SNR) of its channel. In [16] author proposed an Opportunistic Adaptive Routing protocol to exploiting path diversity and select forwarding path opportunistically by focusing on multiple simultaneous flows in wireless mesh networks. In [17] an opportunistic routing protocol was proposed to concentrate on the co-channel interference and the tradeoff between multiplexing and spatial diversity. The proposed protocol is used for multi-channel multi-radio multi-hop wireless networks. 


\section{International Journal of Science and Research (IJSR) \\ ISSN (Online): 2319-7064}

Index Copernicus Value (2013): 6.14 | Impact Factor (2015): 6.391

\section{Methods / Approach}

Our proposed routing method allows a collection of mobile computers which may not be close to any base station and can exchange data along changing and arbitrary paths of interconnection to afford all computers among their number a (possibly multi-hop) path along which data can be exchanged In addition our solution must remain compatible with operation in cases where a base station is available. By the methods outlined here not only will routing be seen to solve the problems associated with ad-hoc networks, but in addition, CSMA/CA is used to control the medium access to avoid collisions in the network.

The Destination-Sequenced Distance-Vector (DSDV) routing algorithm is based on the idea of the classical Bellman-Ford routing algorithm with certain improvements. Every mobile station maintains a routing table that lists all available destinations, the number of hops to reach the destination and the sequence number assigned to the destination node. The sequence number is used to distinguish stale routes from new ones and thus avoid the formation of loops. The stations periodically transmit their routing tables to their immediate neighbors. A station also transmits its routing table if a significant change has occurred in its table from the last update sent. So, the update is both time-driven and event-driven [2].

Each route update packet, in addition to the routing table information, also contains a unique sequence number assigned by the transmitter. The route labeled with the highest (i.e. most recent) sequence number is used. If two routes have the same sequence number then the route with the best metric (i.e. shortest route) is used. Based on the past history, the stations estimate the settling time of routes. The stations delay the transmission of a routing update by settling time so as to eliminate those update that would occur if a better route were found very soon. [6]

The world of today has become quite fast and reliable mainly because of the wireless communication. In our work, we have studied on CSMA/CA and its performance analysis in WLAN. Using RTS/CTS in CSMA/CA protocol is efficient because in the WLAN we would like to get as much higher the transmission to get as possible and lower the collision among the different nodes [18]. In CSMA/CA, when a point or node receives a packet that is to be sent, it checks for a free time period of a channel by which the node can send the packet. In CSMA, a node or devices have to wait for a time interval if it finds a busy or transferring process on the network. The time period is known as a backoff factor. The back-off factor is measure with a back-off counter. When the transmitting channel is clear, the back-off counter reaches to zero and the device starts to send data/packet. And when the transmitting channel is not clear but back-off counter is reaches to zero it means that the process is repeated from the sensing again the channel for a random time.[19]. For avoiding a collision in CSMA/CA, every device can reserve the operating channel for a short period of time. The exchange of two control frames- Request to Send (RTS) and Clear to Send (CTS) are used to avoid the risk of collisions between different nodes in the network. For reserving a channel, first, one device sends RTS frame to the intended destination device. This RTS frame contains the information about reservation period. The other devices reply by a CTS control frame which also contain information about the duration of channel reservation. As the duration of the reservation has been sent in both RTS and CTS, all hosts that could collide with either the sender or the reception of the data frame are informed of the reservation. They can compute the total duration of the transmission and defer their access to the transmission channel until then. [20]

\section{Results / Discussion}

The program is designed and implemented using Microsoft visual studio 2010, where The main interface screen containsa coordination of our map which is (width, height and drawing scale) (figure 1). A list of all other functions of the program is illustrated in (figure2).

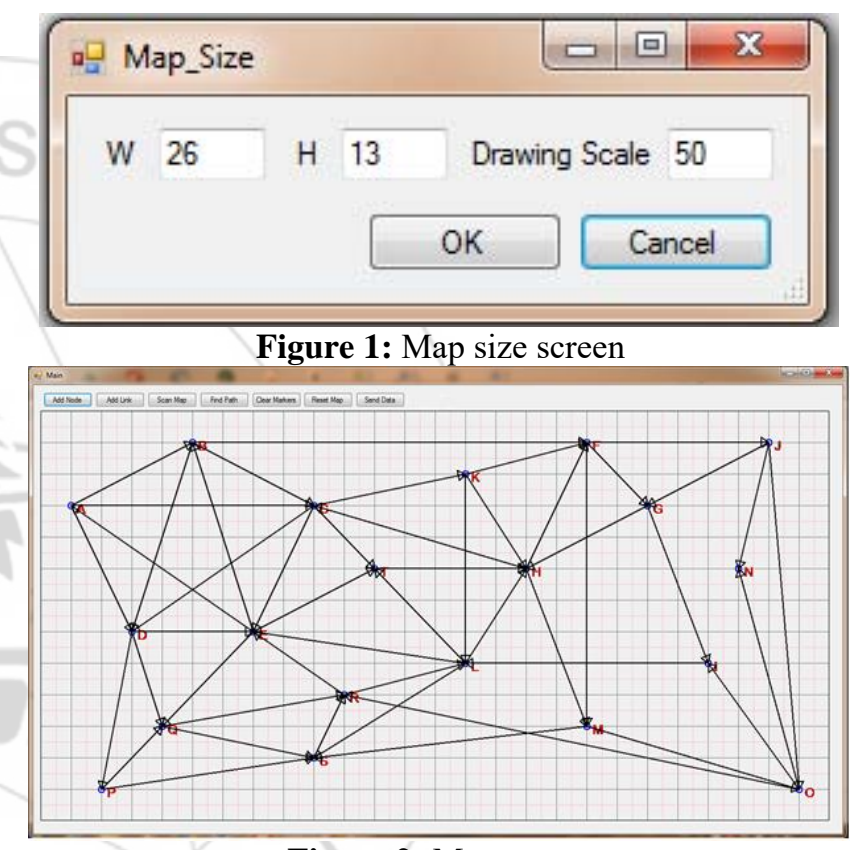

Figure 2: Map screen

Other interfaces are:

1) Add node: the proposed network is scalable where a new node can be added by specifying its $\mathrm{x}$-axis and $\mathrm{y}$-axis coordinates along with a name (letter or character) for the newly added node (figure 3 ).

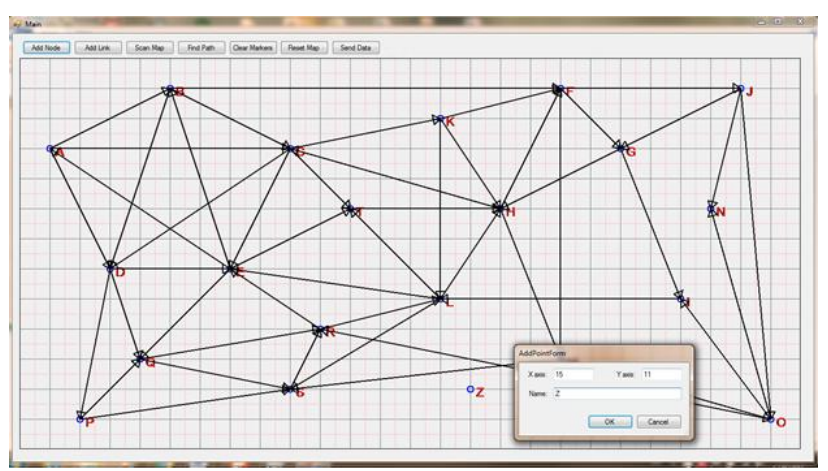

Figure 3: Add node

2) Add link: used to build a connection between any two nodes on the network by choosing first and second nodes respectively from dropping menu (figure 4).

\section{Volume 5 Issue 7, July 2016 www.ijsr.net}




\section{International Journal of Science and Research (IJSR) ISSN (Online): 2319-7064}

Index Copernicus Value (2013): 6.14 | Impact Factor (2015): 6.391

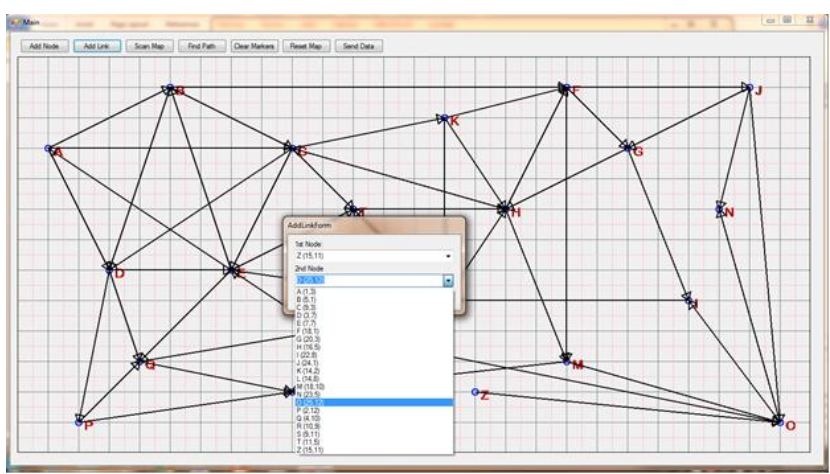

Figure 4: Add link

3) Scan map: it contains a list of all connections between nodes in the network; the word (null) means there is no connection between two specific nodes, (figure 5).

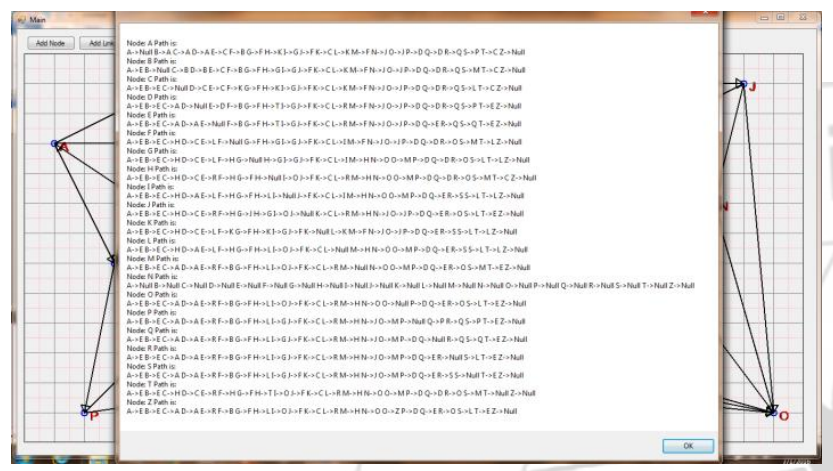

Figure 5: Scan map

4) Find path: applying Destination-Sequenced DistanceVector Routing (DSDV) to find the shortest path between source and destination nodes as discussed in the previous section, (figure 6) and (figure 7).

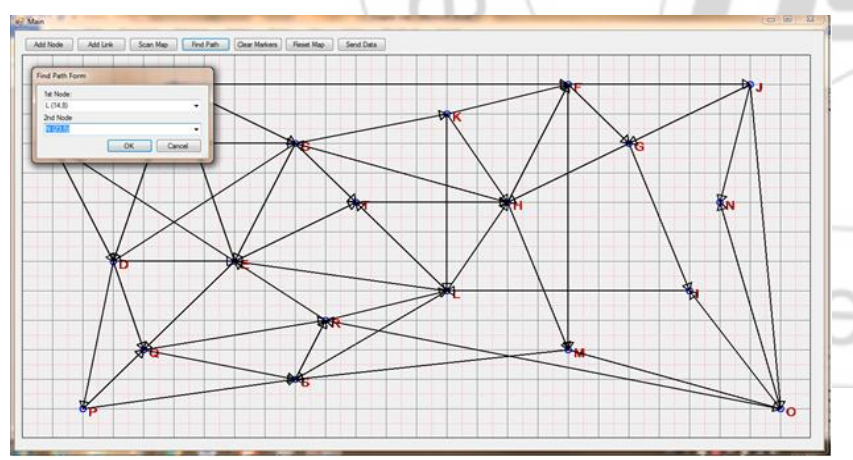

Figure 6: Source and destination nodes

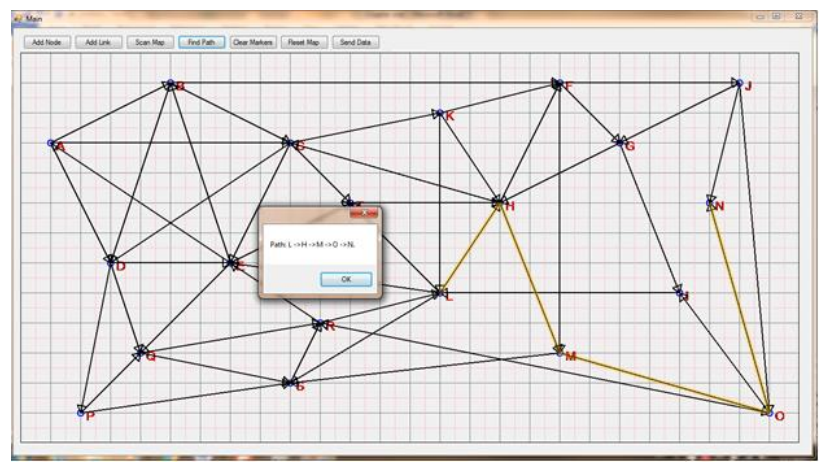

Figure 7: Finding shortest path (yellow line)
6) Reset map: used to delete all added nodes and links to the network.

7) Send data: after finding the shortest path between selected nodes, the path is checked to see if it is idle to send data using CSMA/CA with RTS/CTS mechanism as discussed in the previous section, (figure 8). If the selected path is busy in sending another data, then the alternative path will be checked using the same scenario to transform data packet.

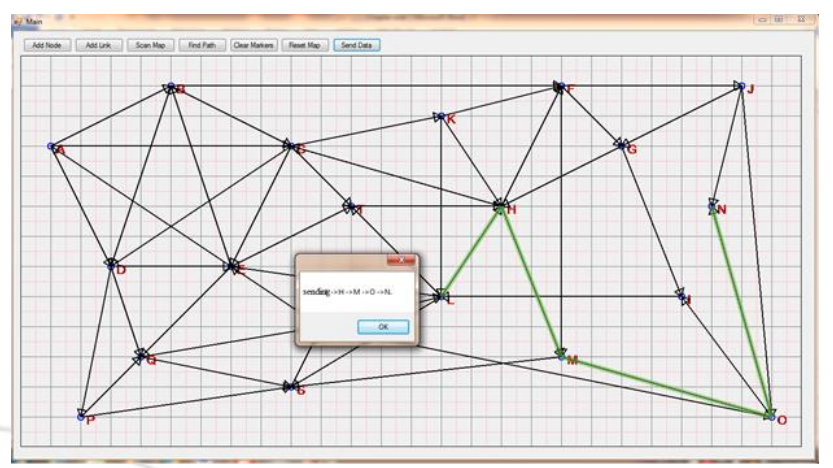

Figure 8: Sending data (green line)

\section{Conclusions}

MANETs gives the user huge number of benefits, so the use of it has grown over the years. Using routing protocols to find an optimal route in ad hoc network is an interesting area of research. This paper focused on the evaluation of DSDV which is a simplest known routing algorithm for ad hoc network that solves loop and counts to infinity problems in distance vector, and to get higher successful transmission rate and lower the collision among nodes CSMA/CA protocol with RTS/CTS mechanism is proposed witch give as an efficient result in sending data and lower packet loss across the network.

\section{Future Scope}

Our future work will focus on evaluating security in DSDV because the user needs the security to send data in the network And optimizing DSDV performance byreducing end to end delay.

\section{References}

[1] T.Wan E. Kranakis P. Oorschot, "Securing the Destination-Sequenced Distance Vector Routing Protocol (S-DSDV)", Proceedings of the 6th International Conference on Information and Communications Security, Malaga, Spain. October 2729, 2004. LNCS vol. 3269, pp.358-374.

[2] M.Reza P. Teymoori, N. Yazdani, et.al "Evaluating Effectiveness of DSDV Routing Protocol on IEEE 802.11n Wireless LANs", International Journal of Electrical \& Computer Sciences IJECS-IJENS Vol: 10 No: 04.

[3] G. Bianchi, "Performance analysis of the ieee 802.11 distributed coordination function", Selected Areas in Communications, IEEE Journal on, vol. 18, no. 3, pp. $535-547$, march 2000.

5) Clear marks: used to delete all colored lines in the map. 


\section{International Journal of Science and Research (IJSR) \\ ISSN (Online): 2319-7064}

Index Copernicus Value (2013): 6.14 | Impact Factor (2015): 6.391

[4] "Ieee 802.11-saturation throughput analysis", Communications Letters, IEEE, vol. 2, no. 12, pp. 318 320, dec. 1998

[5] Baher M. Jean B., et.al. "Multiband CSMA/CA with RTS-CTS Strategy", arXiv: 1501.01430v1 [cs.NI], 7 Jan 2015.

[6] C.E. Perkins \& P. Bhagwat, "Highly Dynamic Destination Sequence-Vector Routing (DSDV) for Mobile Computers", Computer Communication Review, 24(4), 1994, 234-244.

[7] Pei, G, Gerla, M. Chen, T. Fisheye "State Routing: A Routing Scheme for Ad Hoc Wireless Networks". Proceedings of the IEEE International Conference on Communications. 2000.

[8] Vahid G., "Simulating Network traffic in Multi-hop Wireless Ad Hoc Networks based on DSDV", University of Waterloo, Fall 2001.

[9] Lakshmi, M. and Sankarnarayanan, P.E. "Performance analysis of three routing protocols in wireless ad hoc networks". Information technology journal 5(1), 2006 pp 114-120.

[10] Peter, K. Keong L., Hsu W. J., and Yi P. "Performance Evaluation of Efficient and Reliable Routing Protocols for Fixed-Power Sensor Networks". IEEE Transactions On Wireless Communications, Vol. 8,( 5)5, May 2009.

[11] A, N. Maurya, \& N. Gaur, "Performance Evaluation of Mobile Ad Hoc Network Extension to Routing Protocols Simulation Based", VSRD International Journal of Computer Science \& Information Technology, pp.2231-2471, 2(10), 2012.

[12] Lei Z., Deying L. et.al. "Energy-efficient traffic-aware detour trees for geographical routing" International Journal of Computer Networks \& Communications (IJCNC), Vol.2, No.1, January 2010.

[13] Khalil U. Rahman k. et.al. “An Efficient DSDV Routing Protocol for MANET and its usefulness for providing internet Access to Ad Hoc Hosts". Proceedings of the Int'l Conf. of IEEE in ISBN: 978-0-7695-3325-4, 2008.

[14] Nayan R., Laxminath T., et.al. "Analysis and Improvement of DSDV Protocol", IJCSI International Journal of Computer Science Issues, Vol. 8, Issue 5, No 1, ISSN (Online): 1694-0814, September 2011.

[15] Chan-soo H. ;Cioff, J.M. , "Opportunistic CSMA/CA for achieving multi-user diversity in wireless LAN",IEEE Transactions on Wireless Communications, vol.8, no.6, pp.2972-2982, June 2009

[16]Eric R., Jayesh S., et.al. "SOAR: Simple Opportunistic Adaptive Routing Protocol for wireless Mesh Networks", IEEE transactions on Mobile computing, 2009.

[17] Kai Z., Zhenyu Y., et.al.; "OR:Opportunistic Routing in Multi-Radio Multi-Channel Multi-Hop Wireless Networks", IEEE transactions on Wireless Communications, vol.9, Nov 2010.

[18] Muhammad Y, A.K.M Fazlul, et.al. "Performance Analysis of CSMA/CA in Wireless Local Area Network", International Journal of Computer Applications (0975 - 8887) Volume 120 - No.10, June 2015.

[19] C. H. Yeh, "A collision-controlled MAC protocol for mobile ad-hoc networks and multi hop wireless LANs" to be appeared in Proceeding of IEEE Global Telecommunications Conference, 2004.
[20] IEEE 802.11 WG, "Part 11: wireless LAN medium access control (MAC) and physical layer (PHY) specifications: medium access control (MAC) quality of service (QoS) enhancements," IEEE 802.11e D8.0, Feb. 2004.

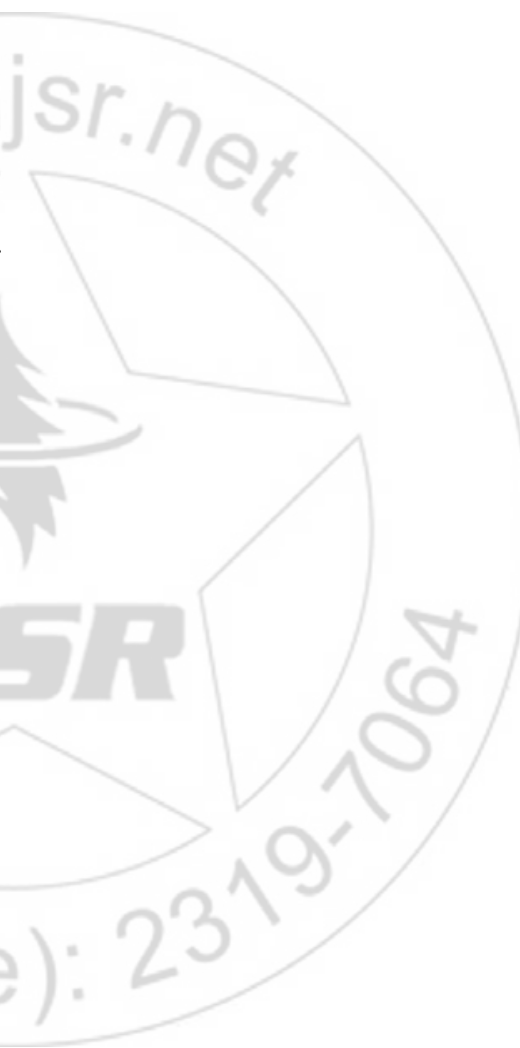

